\title{
The Follicular Distribution and Abundance of Resident Bacteria on Human Skin
}

\author{
By J. N. KEARNEY, ${ }^{1}$ D. HARNBY,${ }^{1}$ G. GOWLAND ${ }^{1 *}$ AND \\ K. T. HOLLAND ${ }^{2}$ \\ University Departments of Immunology ${ }^{1}$ and Microbiology ${ }^{2}$, The General Infirmary, \\ Great George Street, Leeds LSI 3EX, UK
}

(Received 31 October 1983)

\begin{abstract}
A cryostat sectioning procedure was used to determine quantitative viable counts of microorganisms both on the surface and in successive layers of human cadaver skin biopsies. Also, using a previously described xenograft model, we investigated the dependence of microorganisms on the presence of sebaceous glands by using full thickness $(1.5-2.0 \mathrm{~mm})$ and split thickness $(0.6 \mathrm{~mm}$, ensuring sebaceous gland exclusion) human skin. Our results show substantial variation in the distribution and abundance of skin bacteria, even amongst biopsies from the same cadaver. In general, propionibacteria were distributed within a narrow band at varying depths beneath the skin surface whereas staphylococci were more broadly distributed. The importance of this with respect to topically applied antiseptics and antimicrobial agents is indicated. The xenograft studies demonstrated that propionibacteria were dependent on the presence of sebaceous glands whereas staphylococci were not.
\end{abstract}

\section{INTRODUCTION}

There is controversy as to the precise location of bacterial species resident on human skin and the factors affecting their abundance (Noble, 1981). This information is essential for understanding cutaneous microbial ecology and the factors affecting skin disease and treatment. Microscopic studies have demonstrated dense microbial growth both on the skin surface around pilosebaceous follicles, and in the depths of pilosebaceous units (Montes \& Wilborn, 1969; Wolff \& Plewig, 1976). The techniques used in those studies could not differentiate between microbial species, or living and dead cells. Viable sampling procedures have not proved sufficiently sensitive to determine any stratification in follicular distributions (Puhvel et al., 1975; Evans \& Stevens, 1976; Holland et al., 1974). The purpose of this investigation was to develop a cryostat sectioning technique to provide information concerning the distribution of viable bacteria in human skin. In addition, a xenograft model (Kearney et al., 1982a) has been used to determine the dependence of certain organisms on the presence of sebaceous glands.

\section{METHODS}

Human skin. Strips of human cadaver skin were removed from the lateral mid thorax within $36 \mathrm{~h}$ of death. This body region possesses sebaceous follicles in addition to various types of hair follicle (Marples, 1965). The microbiological profile of cadaver skin was previously shown not to differ from that of normal human skin (Kearney et al., 1982a). From these strips a number of $6 \mathrm{~mm}$ diameter biopsies were taken using a sterile biopsy punch. These were snap frozen in a stream of $\mathrm{CO}_{2}$ and subsequently maintained individually in sterile bijou bottles at $-70^{\circ} \mathrm{C}$.

Effect of freezing on skin biopsies. A comparison was made of the microbiological densities in biopsies before and at various times (up to $168 \mathrm{~h}$ ) after snap freezing. The epidermis with attached follicles was separated from the dermis to permit easy homogenization. This separation was achieved by soaking the biopsy at $4{ }^{\circ} \mathrm{C}$ for $2 \mathrm{~h}$ in $1 \mathrm{M}$ $\mathrm{CaCl}_{2}$ thereby loosening dermal/epidermal adhesion (Kellum, 1966). This treatment does not affect the viability of micro-organisms normally resident on human skin (Puhvel et al., 1975). The epidermal portion was ground in a 
micro-tissue homogenizer (Pierce Chemical Co., Ill., USA) in half-strength wash fluid $(0.075 \mathrm{M}$-sodium phosphate buffer, $\mathrm{pH} 7.9$, with $0.1 \%$ Triton X-100; Williamson \& K ligman, 1965) to release micro-organisms. Samples were serially diluted and plated onto appropriate recovery media (Kearney et al., 1982a). Resulting colonies were counted and expressed as $\log _{10}$ (c.f.u. per biopsy). Six biopsies were examined at each time interval.

Treatment of snap frozen biopsies. Frozen biopsies were transferred, without thawing, to the cryostat for sectioning. Transverse sections were cut at a thickness of $20 \mu \mathrm{m}$ and each successive series of 10 sections was pooled to give a sample representing a $0.2 \mathrm{~mm}$ depth of skin. From each biopsy $120.2 \mathrm{~mm}$ samples were obtained. The cryostat knife was sterilized with $70 \%$ ethanol after cutting each sample; residual material was removed with a sterile swab. Each $0.2 \mathrm{~mm}$ sample was homogenized separately in half-strength wash fluid (Williamson \& Kligman, 1965). Homogenates were then serially diluted and plated onto various recovery media (see above).

Skin grafting experiments. The xenograft model described by Kearney et al. $(1982 a, b)$ was used to investigate the dependence of certain bacteria on the presence of sebaceous follicles in human skin. Split thickness $(0.6 \mathrm{~mm})$ and full thickness $(1.5-2.0 \mathrm{~mm})$ skin was taken from each cadaver and the initial microbial densities were determined for each. Histological sections demonstrated that sebaceous glands were excluded in split thickness skin but present in full thickness skin. Grafts $\left(1 \mathrm{~cm}^{2}\right)$ of each thickness were transplanted onto the dorso-lateral thoracic surface of hairless mice, and graft rejection was abrogated by intra-peritoneal administration of anti-mousethymocyte globulin prepared in rabbits (Brent et al., 1967). Bandages were removed at $7 \mathrm{~d}$ post-grafting and the grafts were excised $2 \mathrm{~d}$ later. The epidermal component with attached follicles was processed as described previously, for microbiological assessment.

\section{RESULTS}

Effect of freezing and storage. Table 1 shows a comparison of microbiological densities in biopsies before and at various times after freezing. The results show that this treatment did not adversely affect microbial density.

Distributional data. Figure 1 shows the microbial distributions within a single biopsy. In this case the square-root transformed distributions of both staphylococci and propionibacteria were smooth curves, with the major reservoirs being some distance below the surface within the pilosebaceous canals. The propionibacteria exhibited a narrower distribution than the staphylococci.

Schematic representations of the observed distributional patterns and their frequencies amongst all biopsies, for both staphylococci and propionibacteria, are shown in Fig. 2. The results demonstrate that the surface-located micro-organisms were generally only a small proportion of the total viable skin population, although distributional patterns varied.

The breadth of distribution of each genus was investigated in greater detail by calculating an evenness index (niche breadth index). The inverse of Simpson's index (Simpson, 1949: Levins, 1968) was used, where $B=1 / \Sigma \mathrm{p} i^{2}$ and $\mathrm{p} i=$ proportion of microbes in sample $i$. With 12 samples within a biopsy this would give a theoretical maximum $B$ of $12 \cdot 0$, where bacteria were completely evenly distributed, and a minimum $B$ of 1.0 , where all bacteria were concentrated within a single sample. The mean evenness index and $95 \%$ confidence limits for each genus in the biopsies studied were: staphylococci, $B=5.8 \pm 1.08$ (16 biopsies) and propionibacteria $B=3.3 \pm 1.07$ (14 biopsies), indicating that the staphylococci were much more evenly distributed. The narrower distribution of propionibacteria is probably due to the reduced range

\section{Table 1. Effects of snap freezing and storage of biopsies at $-70^{\circ} \mathrm{C}$ on viability of micro-organisms}

The results are for six biopsies at each time interval and represent mean $\log _{10}$ (c.f.u. per biopsy) $\pm 95 \%$ confidence limits.

\begin{tabular}{|c|c|c|c|c|c|}
\hline Organism & $\begin{array}{r}\text { Time at } \\
-70^{\circ} \mathrm{C}(\mathrm{h})\end{array}$ & 0 & 1 & 24 & 168 \\
\hline i & & $4.29 \pm 0.54$ & $4 \cdot 12 \pm 0.38$ & $4.09 \pm 0.79$ & $4 \cdot 32+0.86$ \\
\hline cteria & & $3.45 \pm 0.95$ & $3.36 \pm 0.98$ & $3.59 \pm 0.98$ & $4.06 \pm 0.98$ \\
\hline erobic coryneforms & & $3.82 \pm 0.83$ & $3.56 \pm 0.83$ & $3.73 \pm 0.92$ & $4.01 \pm 0.95$ \\
\hline
\end{tabular}




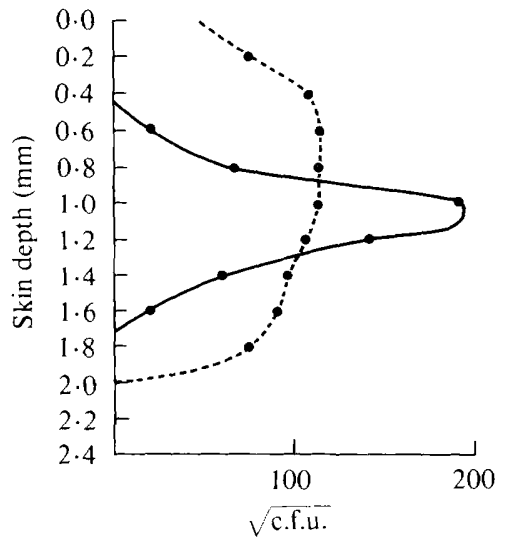

Fig. 1. Microbial distributions within a single $0.6 \mathrm{~mm}$ diameter biopsy of human cadaver mid-thoracic skin. ----, Staphylococci; —_, propionibacteria.

(a) Pattern of microbial distribution:

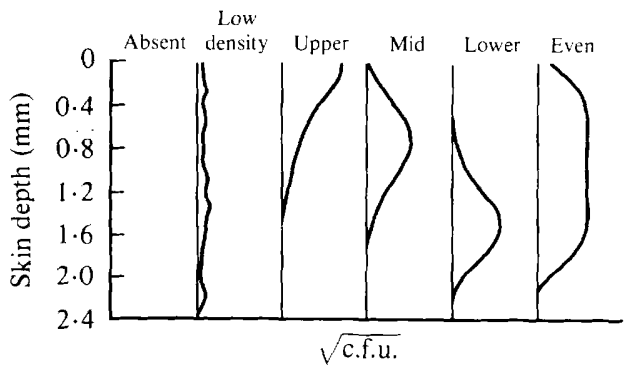

(b) Frequency of occurrence:

\begin{tabular}{rl|l|l|l|l|l} 
Staphylococci & 7 & 3 & 4 & 3 & 0 & 6 \\
Propionibacteria & 9 & 4 & 2 & 6 & 2 & 0
\end{tabular}

Fig. 2. (a) Schematic representation of the patterns of microbial distributions within the total sample of $0.6 \mathrm{~mm}$ diameter human cadaver mid-thoracic skin biopsies studied: $(b)$ the frequency of pattern occurrence for each bacterial genus.

of environmental conditions under which the more fastidious propionibacteria are able to grow. The broader staphylococcal distribution was not due to differences in distribution of individual Staphylococcus species. None of the Staphylococcus or Propionibacterium species exhibited specific distributional patterns.

Xenograft experiments. The density of staphylococci on both split and full thickness grafts was not significantly different from the density before grafting. In contrast, the density of propionibacteria on split thickness grafts decreased significantly, whereas full thickness skin maintained a normal carriage (Fig. 3). Further experiments showed no decrease in propionibacteria on split thickness grafts at $1 \mathrm{~d}$ post-grafting, demonstrating that the decline was not due to an acute environmental effect resulting from the grafting procedure. The most likely explanation is that propionibacteria have an absolute requirement for sebaceous gland products for their continued growth and survival whereas staphylococci do not. 

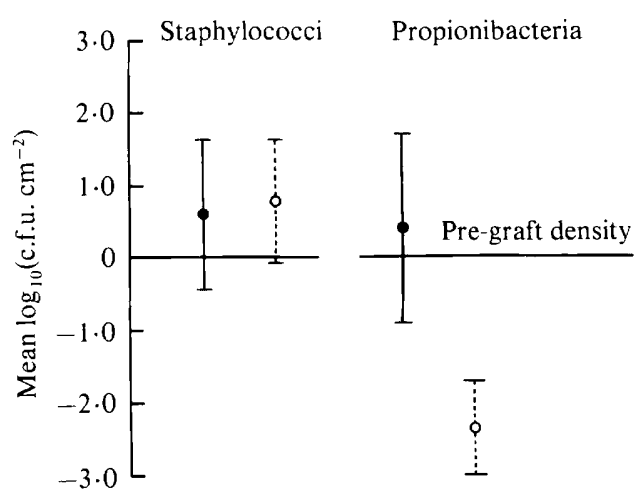

Fig. 3. Change in bacterial density on human mid-thoracic skin $9 \mathrm{~d}$ after transplantation to hairless mice. $0,0.6 \mathrm{~mm}$ thick skin (no sebaceous glands);, $1.5-2.0 \mathrm{~mm}$ thick skin (intact sebaceous glands). The bars represent $95 \%$ confidence limits.

\section{DISCUSSION}

To summarize the distributional data, this study does not support the conventional view of staphylococci being located primarily in the upper regions of pilosebaceous follicles with propionibacteria underlying them. Our data for thoracic skin suggest a more heterogeneous situation, where both genera may be found in high densities in all parts of the follicular canal. With regard to skin disinfection, these results indicate that the complete elimination of either genus would require the penetration of disinfectant throughout the whole pilosebaceous canal, as demonstrated experimentally by Selwyn \& Ellis (1972). This applies not only to prophylactic skin disinfection for surgical or aseptic manipulations but also to the use of topical antibiotics in disease states such as acne vulgaris.

No significant differences in the density of staphylococci and propionibacteria were detected by analysis of variance amongst cadavers. This was due to the large variation in density amongst biopsies from the same individual. This in turn reflects the heterogeneity in follicular colonization recently demonstrated by J. P. Leeming (personal communication) who showed that less than $50 \%$ of normal follicles were colonized by micro-organisms. This is consistent with our findings that many $6 \mathrm{~mm}$ thoracic cadaver skin biopsies, containing an average of 6 follicles, did not yield any micro-organisms. The factors pre-disposing a follicle to microbial colonization are not known. This result, however, suggests that data expressing microbial density as numbers per unit area are not very meaningful. High densities may result from a high frequency of follicular colonization, or from high densities within only a small proportion of follicles.

The dependence of cutaneous micro-organisms on sebaceous gland products is also contentious. Cove et al. (1980 a) could find no correlation in man between sebum excretion rate and density of propionibacteria, whereas Kearney et al. (1984) discovered a small positive correlation in two independent populations. Administration to patients of 13-cis-retinoic acid, which is known to cause a cessation of sebaceous gland excretion, resulted in a dramatic decrease in propionibacteria, whereas staphylococcal density was relatively little affected (King et al., 1982).

A correlation has also been discovered between the presence of triglycerides in mammalian sebum and the carriage of propionibacteria, but not of staphylococci (Webster et al., 1981). Our experiments indicate that propionibacteria but not staphylococci are dependent on the presence of sebaceous glands, or their products. Triglycerides or their cleavage products seem likely candidates, although in view of the low correlation between density of propionibacteria and sebum excretion rate it is unlikely to be the growth limiting nutrient. One cleavage product of triglycerides, oleic acid, has been shown to substitute for the vitamin biotin in the nutrition of propionibacteria (Holland et al., 1979); however, staphylococci also require this vitamin and also demonstrate oleic acid as a replacement (Cove et al., 1980 b). Confirmation of the specific requirement in vivo remains to be established. 


\section{REFERENCES}

Brent, L., Courtenay, T. \& Gowland, G. (1967). Immunological reactivity of lymphoid cells after treatment with anti-lymphocytic serum. Nature, London 215, 1461-1464.

Cove, J. H., Holland, K. T. \& Cunliffe, W. J. $(1980 a)$. An analysis of sebum excretion rate, bacterial population and the production of free fatty acids on human skin. British Journal of Dermatology 103, 383-386.

Cove, J. H., Holland, K. T. \& Cunliffe, W. J. $(1980 \mathrm{~b})$. The vitamin requirements of staphylococci isolated from human skin. Journal of Applied Bacteriology 49, 29-37.

Evans, C. A. \& Stevens, R. J. (1976). Differential quantitation of surface and subsurface bacteria of normal skin by the combined use of the cotton swab and scrub methods. Journal of Clinical Microbiology 3. 576- 581 .

Holland, K. T., Roberts, C. D., CunlifFe, W. J. \& Williams, M. (1974). A technique for sampling microorganisms from the pilosebaceous ducts. Journal of Applied Bacteriology 37, 289-296.

Holland, K. T., Greenman, J. \& Cunliffe, W. J. (1979). Growth of cutaneous propionibacteria on synthetic medium: growth yields and exoenzyme production. Journal of Applied Bacteriology 47, 383394.

Kearney, J. N., Gowland, G., Holland, K. T. \& CUNliffe, W. J. (1982a). Transplantation model for study of microbiology of human skin. Lancet ii, 334 335 .

Kearney, J. N., Gowland, G., Holland, K. T. \& Cunliffe, W. J. (1982b). Maintenance of the normal flora of human skin grafts transplanted to mice. Journal of General Microbiology 128, 24312437.

Kearney, J. N., Ingham, E., Cunliffe, W. J. \& HollaNd, K. T. (1984). Correlations amongst human skin variables of medical and ecological importance. British Journal of Dermatology (in the Press).

KeLluM, R. E. (1966). Isolation of human sebaceous glands. Archives of Dermatology 93, 610-612.

King, K., Jones, D. H., Daltrey, D. C. \& Cunliffe, W. J. (1982). A double-blind study of the effects of 13-cis-retinoic acid on acne, sebum excretion rate and microbial population. British Journal of Dermatology 107, 583-590.

LEVINS, R. (1968). Evolution in Changing Environments. Princeton, NJ: Princeton University Press.

Marples, M. J. (1965). The Ecology of Human Skin. Springfield: C. C. Thomas.

Montes, L. F. \& WILBORN, W. H. (1969). Location of bacterial skin flora. British Journal of Dermatology 81, suppl. 1, 23-26.

Noble, W. C. (1981). Microbiology of Human Skin. 2nd edn. London: Lloyd-Luke.

Puhvel, S. M., Reisner, R. M. \& Amirian, D. A. (1975). Quantification of bacteria in isolated pilosebaceous follicles in normal skin. Journal of Investigative Dermatology 65, 525-531.

Selwyn, S. \& Ellis, H. (1972). Skin bacteria and skin disinfection reconsidered. British Medical Journal 1, 136-140.

Simpson, E. H. (1949). Measurement of diversity. Nature, London 163, 688.

Webster, G. F., RugGieri, M. R. \& MCGinley, K. J. (1981). Correlation of Propionibacterium acnes population with the presence of triglycerides on nonhuman skin. Applied and Environmental Microbiology 41, 1269-1270.

Williamson, P. \& Kligman, A. M. (1965). A new method for the quantitative investigation of cutaneous bacteria. Journal of Investigative Dermatology 45, 498-503.

Wolff, H. H. \& Plewig, G. (1976). Ultrastruktur der Mikroflora in Follikeln und Komedonen. Hautarzt 27, 432-440. 